\title{
Insomnia and excessive daytime sleepiness in obstructive sleep apnea: only different clinical phenotypes?
}

\author{
Charalampos Mermigkis ${ }^{1,2,3} \cdot$ Izolde Bouloukaki $^{1}$ - Sophia E. Schiza ${ }^{1}$
}

Received: 6 March 2015 / Revised: 19 March 2015 / Accepted: 25 March 2015 / Published online: 9 April 2015

(C) Springer-Verlag Berlin Heidelberg 2015

Insomnia and excessive daytime sleepiness represent two important public-health problems that require accurate diagnosis and effective treatment. Obstructive sleep apnea (OSA) is highly prevalent [1] and may be related to both excessive daytime sleepiness (EDS) and insomnia.

Several studies have shown that OSA is associated with EDS that represents a frequent and serious symptom of the syndrome [2,3]. On the other hand, other studies $[4,5]$ report that many OSA patients, including some patients with severe OSA, do not experience excessive sleepiness. The sleepy and non-sleepy phenotypes are now commonly used terms in OSA [6]. When suspicion of OSA exists, physicians should not defer a sleep study for patients simply because they deny excessive daytime sleepiness by clinical history or score low on the Epworth sleepiness scale (ESS). Insomnia is another sleep disorder commonly associated with obstructed breathing during sleep, but this association has received less focus compared to excessive daytime sleepiness [7, 8]. Inconsistencies in the definitions and assessments of insomnia across studies hinder our understanding of the prevalence of co-morbid insomnia in OSA and limit the ability to compare findings across studies. Taking all the above into account, a recent review [7] reported insomnia to be present in $39-58 \%$ of patients with OSA. In addition, the criteria for the insomnia

Charalampos Mermigkis

mermigh@gmail.com

1 Sleep Disorders Unit, Department of Thoracic Medicine, University General Hospital, Medical School of the University of Crete, Crete, Greece

2 Sleep Disorders Center, 401 General Army Hospital, Thrakis 61A, Vrilissia, Athens, Greece

3 Sleep Disorders Center, Henry Dunant Hospital, Athens, Greece diagnosis were recently changed, both in the Diagnostic and Statistical Manual for Mental disorders (DSM-V) [9] and in the International Classification of Sleep Disorders-3 (ICSD-3) [10]. The main change is that non-restorative sleep is no longer part of the insomnia diagnosis in DSM-V/ICSD-3. Nonrestorative sleep is reported to be more common in OSA patients than problems with sleep initiation and maintenance [7, 8]. Hence, the new insomnia criteria will probably reduce the reported prevalence of insomnia in OSA patients.

In 1973, Guilleminault et al. [11] described the association between insomnia and obstructive sleep apnea, and called it "sleep-insomnia apnea syndrome." The relationship between these two common sleep disorders is complex and in many points unclear. Based on published studies, there is a higher incidence of breathing disorders in insomniac patients than there is in the general population [7, 8, 12]. Lichstein et al. [13] demonstrated that high proportions of individuals, particularly the elderly, present this combined condition of undiagnosed sleep apnea and insomnia. Insomnia is primarily diagnosed clinically with a detailed medical, psychiatric, and sleep history. Polysomnography (PSG) is indicated when a sleeprelated breathing disorder or periodic limb movement disorder is suspected, initial diagnosis is uncertain, treatment fails, or precipitous arousals occur with violent or injurious behavior. However, PSG is not indicated for the routine evaluation of transient insomnia, chronic insomnia, or insomnia associated with psychiatric disorders [14].

In this issue of Sleep and Breathing [15], Bjørn Bjorvatn et al. report their results related to the prevalence of insomnia and excessive sleepiness in relation to the presence and severity of OSA. The large included sample consisted of $1115 \mathrm{pa}-$ tients referred to a university hospital in Norway on suspicion of OSA. Obstructive sleep apnea was diagnosed and categorized based on a standard respiratory polygraphic sleep study using a type 3 portable monitor. The majority of included 
patients had an unattended home sleep study. Validated questionnaires based on standard criteria were used for the insomnia diagnosis (Bergen insomnia scale, BIS) as well as for excessive sleepiness (Epworth sleepiness scale). The Bergen insomnia scale permits diagnosing insomnia using both old and new (from 2014) criteria. Logistic and linear regression analyses were used in order to investigate the prevalence of sleepiness and insomnia according to the severity of OSA based on the apnea-hypopnea index (AHI).

The prevalence of excessive sleepiness was clearly related to OSA severity. More specifically, a clear increase was reported related to OSA severity with percentages of EDS 46.5 and $58 \%$ in mild and severe OSA, respectively. In agreement with previous studies, there were also patients with severe OSA and no EDS based on the ESS.

Insomnia on the other hand was not significantly higher in prevalence depending on OSA severity, neither based on the old nor the new 2014 diagnostic criteria. Using the old criteria, the prevalence of insomnia was $74-79 \%$, whereas the prevalence using the 2014 criteria was 44-54\%, with lowest value in severe OSA. Based on the new criteria, in which nonrestorative sleep is no longer part of the insomnia diagnosis, percentages of insomnia were 54.9 and $44.6 \%$ in the mild and severe OSA group, respectively. The authors conclude that their study is the first to show that excessive sleepiness is positively associated, whereas insomnia is negatively associated with OSA severity, using validated questionnaires within the same patient population. In addition, it is noteworthy that this is the first study to investigate the prevalence of insomnia in OSA patients with the new 2014 criteria.

The limitations of the study provide direction for further research in this field. Sleep was assessed with home studies using type 3 recording and not with attended in lab polysomnography. Severity was assessed by the AHI that may be underestimated in portable home studies compared to formal PSG [16]. AHI remains a valuable marker of OSA severity but has limitations since other markers such as oxygen desaturation index, mean and nadir oxygen saturation, arousal index, cardiac arrhythmias, presence of hypercapnia, alterations in sleep macro-architecture, etc. may also be important in the characterization of the severity of OSA. The severity of OSA should be evaluated by multiple PSG parameters as well as factors related to clinical history (severity of symptoms, co morbid respiratory, cardiac, neurologicpsychiatric disorders, etc.) and not only with the AHI. It will be interesting to investigate if the prevalence of insomnia has the same characteristics (namely, decreased percentages in severe OSA) when other parameters alone or in association with the AHI are used in order to assess OSA severity.

Another crucial point related to insomnia in patients with OSA is the occurrence other respiratory and cardiac comorbidities such as cardiac failure, respiratory diseases with obstructive or restrictive pattern such as chronic obstructive pulmonary disease (COPD) or idiopathic pulmonary fibrosis (IPF) that are also characterized by the disabling symptom of insomnia irrespective of OSA coexistence $[17,18]$. In addition, insomnia may have direct or indirect effects on CPAP adherence in these patients. Patients with OSA and insomnia may have more difficulty adhering to CPAP because of increased awareness of the mask due to frequent awakenings and an inability to initiate or return to sleep with the mask in place. Psychiatric disorders, such as anxiety and depression, often coexist with insomnia and may also play a role in CPAP adherence. For example, anxious patients or patients with specific types of breathing during sleep [19] may be more likely to have claustrophobia, which is associated with poor CPAP adherence. Based on the findings of the current study, insomnia appears to characterize mainly mild to moderate OSA. The main question is then, how can treating physicians deal with the multiple CPAP non-adherence issues that are commonly seen in such patients? The last may only be achieved in a wellorganized CPAP clinic where intense follow-up and physiologic support as well as pharmacologic approaches may be applied [20]. For example nonbenzodiazepine agents have hypnotic and sedative effects similar to those of benzodiazepines, but some may have fewer muscle-relaxant effects, thus offering a more favorable treatment approach to insomnia in OSA.

In conclusion, the study by Bjørn Bjorvatn is the first to show that excessive sleepiness is positively associated with OSA severity, whereas insomnia (especially based on the new 2014 criteria) is negatively associated with OSA severity, using validated questionnaires within the same patient population. Indeed, further studies are needed to investigate if the role of PSG should be re-evaluated in patients with OSA and co-morbid insomnia. Currently, PSG is not routinely used in insomnia but it may be that PSG can play a significant role both in characterizing the diagnoses and directing treatment.

\section{References}

1. Young T, Peppard PE, Gottlieb DJ (2002) Epidemiology of obstructive sleep apnea: a population health perspective. Am J Respir Crit Care Med 165(9):1217-1239

2. Engleman HM, Douglas NJ (2004) Sleep. 4: sleepiness, cognitive function, and quality of life in obstructive sleep apnoea/hypopnoea syndrome. Thorax 59(7):618-622

3. Sauter C, Asenbaum S, Popovic R, Bauer H, Lamm C, Klösch G, Zeitlhofer J (2000) Excessive daytime sleepiness in patients suffering from different levels of obstructive sleep apnoea syndrome. $\mathrm{J}$ Sleep Res 9(3):293-301

4. Kapur VK, Baldwin CM, Resnick HE, Gottlieb DJ, Nieto FJ (2005) Sleepiness in patients with moderate to severe sleep-disordered breathing. Sleep 28(4):472-477

5. Bixler EO, Vgontzas AN, Lin HM, Calhoun SL, Vela-Bueno A, Kales A (2005) Excessive daytime sleepiness in a general population sample: the role of sleep apnea, age, obesity, diabetes, and depression. J Clin Endocrinol Metab 90(8):4510-4515 
6. Roure N, Gomez S, Mediano O et al (2008) Daytime sleepiness and polysomnography in obstructive sleep apnea patients. Sleep Med 9: $727-731$

7. Luyster FS, Buysse DJ, Strollo PJ Jr (2010) Comorbid insomnia and obstructive sleep apnea: challenges for clinical practice and research. J Clin Sleep Med 6(2):196-204

8. Al-Jawder SE, Bahammam AS (2012) Comorbid insomnia in sleep-related breathing disorders: an under-recognized association. Sleep Breath 16(2):295-304. doi:10.1007/s11325-011-0513-1, Review

9. Association. AP (2013) Diagnostic and statistical manual of mental disorders (DSM-V). American Psychiatric Association, Washington

10. Medicine AAoS (2014) The international classification of sleep disorders, 3nd ed.: diagnostic and coding manual. American Academy of Sleep Medicine, Darien

11. Guilleminault C, Edridge F, Dement W (1973) Insomnia whit sleep apnea: a new syndrome. Science 181:856-858

12. Bjorvatn B, Pallesen S, Gronli J, Sivertsen B, Lehmann S (2014) Prevalence and correlates of insomnia and excessive sleepiness in adults with obstructive sleep apnea symptoms. Percept Mot Skills 118(2):571-586

13. Lichstein K, Reidel B, Lester K, Aguillard R (1999) Occult sleep apnea in a recruited sample of older adults with insomnia. J Consult Clin Psychol 67:405-410

14. Littner M, Hirshkowitz M, Kramer M, Kapen S, Anderson WM, Bailey D, Berry RB, Davila D, Johnson S, Kushida C, Loube DI, Wise M, Woodson T (2003) Sleep: practice parameters for using polysomnography to evaluate insomnia: an update. J Sleep Sleep Disord Res 26(6):754-760

15. Bjorvatn B, Lehmann S, Gulati S, Aurlien H, Pallesen S, Saxvig IW (2015) Prevalence of excessive sleepiness is higher whereas insomnia is lower with greater severity of obstructive sleep apnea. Sleep Breath. doi:10.1007/s11325-015-1155-5

16. Nerfeldt P, Aoki F, Friberg D (2014) Polygraphy vs. polysomnography: missing osas in symptomatic snorers - a reminder for clinicians. Sleep Breath 18(2):297-303. doi:10.1007/ s11325-013-0884-6

17. Mermigkis C, Kopanakis A, Foldvary-Schaefer N, Golish J, Polychronopoulos V, Schiza S, Amfilochiou A, Siafakas N, Bouros D (2007) Health-related quality of life in patients with obstructive sleep apnoea and chronic obstructive pulmonary disease (overlap syndrome). Int J Clin Pract 61:207-211

18. Mermigkis C, Bouloukaki I, Antoniou K, Papadogiannis G, Giannarakis I, Varouchakis G, Siafakas N, Schiza SE (2015) Obstructive sleep apnea should be treated in patients with idiopathic pulmonary fibrosis. Sleep Breath 19(1):385-391. doi:10.1007/ s11325-014-1033-6

19. Mermigkis C, Mermigkis D, Varouchakis G, Schiza S (2012) CPAP treatment in patients with idiopathic pulmonary fibrosis and obstructive sleep apnea-therapeutic difficulties and dilemmas. Sleep Breath 16(1):1-3

20. Bouloukaki I, Giannadaki K, Mermigkis C, Tzanakis N, Mauroudi E, Moniaki V, Michelakis S, Siafakas NM, Schiza SE (2014) Intensive versus standard follow-up to improve continuous positive airway pressure compliance. Eur Respir J 44(5):1262-1274 